\title{
Impact of Load Dynamics on Torsional Interactions
}

\author{
Papiya Dattaray, Peter Wall, \\ V Terzija \\ The University of Manchester \\ Manchester, United Kingdom
}

\author{
Diptargha Chakravorty \\ Electrical Engineering Department \\ Imperial College London, \\ London, United Kingdom
}

\author{
Priyanka Mohapatra, \\ James $\mathrm{Yu}$ \\ S.P. Energy Networks \\ Blantyre, United Kingdom
}

\begin{abstract}
This paper evaluates the impact of load dynamics on torsional interactions by considering a mix of static and dynamic loads aggregated at the bulk transmission level. This is essential to investigate the importance of detailed load modelling for subsynchronous resonance (SSR) studies to accurately assess damping contribution and capture system dynamics. SSR interaction with dynamic loads is investigated for both direct on line and drive connected motor loads. Damping contribution from dynamic loads is also assessed based on their location and size. The interaction of dynamic loads with classical SSR phenomenon is observed and introduced as the new concept of (Subsynchronous Resonance Load Interactions (SSR-LI)). SSRLI assumes critical importance for scenarios where the load and generation centers are in close electrical proximity and impact of loads on torsional damping is significant. Finally, the scope for using existing converter interfaced motors for torsional mode damping has been discussed.
\end{abstract}

Index Terms - Dynamic load, induction motor, static load, subsynchronous resonance (SSR), variable frequency drive.

\section{INTRODUCTION}

Subsynchronous resonance (SSR) is a major concern for the integrity and operation of turbine-generators connected to a transmission system employing series capacitors. For analysis of SSR, computer models of the power system in electromagnetic transient simulations (EMTs) generally focus on the turbines, generators, speed governors and excitation systems. The network is modeled in detail using algebraic and ordinary differential equations; however, the effect of load models is either neglected (IEEE First and Second Benchmark models) or constant impedance models are used [1]. This paper demonstrates the importance of load modelling for SSR studies by simulating the impact of different load models on the torsional interactions, using EMT program. Static load models, like constant impedance type, are found to be fairly inadequate for capturing load and/or system dynamics/transients (suitable only for instantaneous responses) [2, 3]. Dynamic load models, on the other hand, being described by differential equations exhibit timedependent responses that can capture dynamic responses pertaining to exchange of energy between the system and load (e.g. during SSR). For systems having high percentage of induction motor type loads, detailed dynamic load modelling is of utmost importance [4]. The impact of loads on system dynamics has been widely investigated for the classical stability studies [5] but not for torsional dynamics. [6-8]

This work was funded under the Network Innovation Competition in Great Britain as part of the Scottish Power Energy Networks led VISOR project discuss the effect of different load characteristics on transient stability while [9].deals with dynamic stability, considering mostly a mix of static and composite load models.

Load modelling for assessment of SSR becomes critically important when the load center is in close electrical proximity to a generation center [5]. It is worth analyzing if approximate load representations can influence the behavior of RLC resonant circuits. The impact of various load models on the damping characteristics of torsional modes has been observed in [10] for the IEEE First Benchmark Model with linearized models for static and dynamic loads. The linearized analysis neglects generator stator transients and holds valid only for small disturbances (with the assumption of a balanced system model). However, transient torque type of SSR is sparked by large transient system disturbances (e.g. short-circuit faults) that can be studied using EMTs only with detailed non-linear system modelling. Moreover, the linearized analysis fails to capture any non-linear dynamic interactions that might exist between dynamic motor loads and generators under instances of SSR (interactions between motor and generator rotors).

Transient interactions may cause both voltage and a frequency oscillation to appear at the terminals, so modelling of frequency characteristics of loads close to generators is of critical importance. With advanced computational capabilities, there is little reason to represent overall system load equivalents with static models.

The paper looks at several combinations of static and dynamic load models aggregated at the transmission level, shown in Fig. 1, using a kVA weighted averaging technique [4] and demonstrates how their modelling influences the damping of torsional oscillations for a classical case of SSR. Dynamic interaction of the motor loads (both direct on-line and variable frequency drive) has been discussed in particular detail in order to demonstrate how sub-synchronous oscillations propagate through power electronic drive interfaces and affect motor operation. Furthermore, damping contribution from dynamic loads has been evaluated based on motor size and location. The study reveals the impact of load types and their locations on torsional mode damping and illustrates the need for accurate load modelling for precise assessment of SSR risks. The paper also highlights the scope for use of variable frequency drive (VFD) motor loads as auxiliary torsional oscillation damper with subtle modifications to the existing drive controls. 


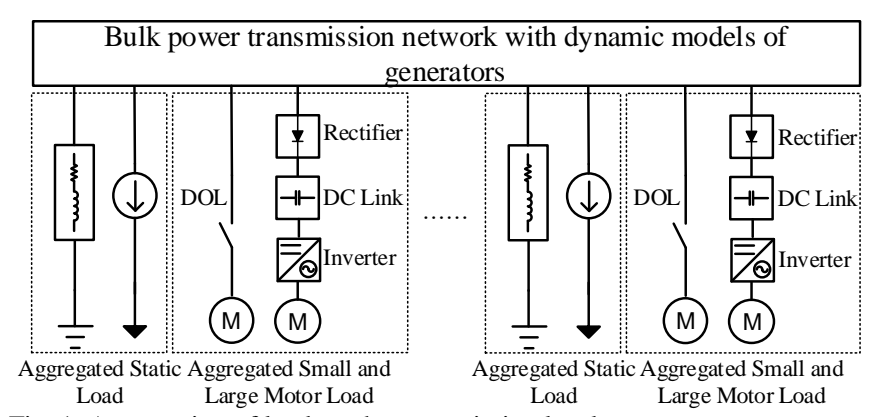

Fig. 1. Aggregation of loads at the transmission level

\section{LOAD MODELLING}

Load modelling is a well-established aspect of power system research. However, much of this research has focused on the modelling of loads for RMS system studies (not EMT) [11] and the model parameter estimation for these wellestablished RMS models [12]. This section outlines the modelling required to represent static and dynamic loads in EMT simulations for the study of SSR.

\section{A. Definition of a Load Model}

Load models approximate the effects of sub-transmission and distribution system lines, cables, reactive power compensation, on load tap changing transformers (OLTC), distribution voltage regulators, and even relatively small synchronous or induction generators [2]. Static load models express the active and reactive powers at any particular instant of time as functions of the bus voltage magnitude and frequency at the same instant. It is used primarily for modelling of static load components (e.g. heating loads, lighting loads, approximation of motor loads etc.). However, dynamic load models express the active and reactive powers at any instant of time as functions of the voltage magnitude and frequency at the past instants using difference or differential equations [2]. In SSR studies the system loads have always been represented by static models (constant impedance type) while assessing risks or studying interactions through EMTs. The following section discusses the different load models used in the current research to study their impact on torsional oscillation damping.

\section{B. Modelling Of Considered Load Types}

In the absence of accurate load data, international industry practice is to represent loads as exponential static models for dynamic studies. The aggregated load is usually represented by constant current type for active power and constant impedance type for reactive power [13]. It is very difficult to correctly assess the exact composition of static and dynamic loads in a network as it may vary with time of the day or changing seasons. About $70 \%$ of the system operators around the world use static load models for power system stability studies while the rest use induction motors to represent dynamic loads [13]. The different types of load models used for this study are discussed in detail in this section.

a) Constant impedance load: For EMT simulations, DigSILENT models lumped loads as passive elements (RLC) either in the form of purely inductive or a mixed inductivecapacitive load. The admittance values are calculated based on the load flow solution. This type of load model represents the constant impedance part of static component of the aggregated power system load at transmission level.The General Load Type (of DigSILENT) with series RL configuration has been used here for representing the constant impedance type load. The same load (in terms of the active and reactive power) can also be represented in the form of a parallel RL configuration. Both configurations were tested for their relative impact on torsional oscillations and the parallel $\mathrm{RL}$ was found to provide more damping. Therefore, only the series configuration is studied in detail here, as it is the worst case.

b) Constant current load: In the absence of exponential or polynomial load models in DigSILENT for EMT simulation, the static load model for constant current type was realized using a static generator with a built-in current controller. The controlled current source model of the static generator is modeled in DigSILENT using a voltage source with current control.

c) Direct on-line (DOL) motor load: The majority of dynamic loads in the industrial and commercial sector are induction motors. These loads can be further classified based on their areas of application e.g. space heating, ventilation, industrial motors, compressed air and refrigeration. Industrial motor has the largest share among motor type loads [14] with sizes ranging from $5 \mathrm{HP}$ to $200 \mathrm{HP}$ and above. In the absence of further classification, this category is assumed to be divided equally into large and small industrial motors.

For EMT simulation of aggregated three phase induction motor loads, models with a single rotor circuit in each axis is generally sufficient (third order model with slip and d, q axis internal voltage or flux as state variables) for large scale simulations [5]. The DigSILENT model for asynchronous machine (double-cage preferred for starting or other kind of transient study) is sixth order and is appropriate for this study.

The generalized model recommended for mechanical load speed-torque characteristic is given in [15]. This torque model, as in (1) and (2), is suitable for a wide range of motor types including large and small industrial motors. The corresponding values for the coefficients are available in [15], these suggest that the bulk of industrial motors are centrifugal type i.e. with a quadratic speed-torque characteristic.

$$
\begin{gathered}
\frac{T_{m}}{T_{m 0}}=A\left(\frac{w_{m}}{w_{m 0}}\right)^{2}+B\left(\frac{w_{m}}{w_{m 0}}\right)+C+D\left(\frac{w_{m}}{w_{m 0}}\right)^{a m} \\
C=1-(A+B+D)
\end{gathered}
$$

d) Variable Frequency Drive (VFD) motor load: Some industrial motors are equipped with variable speed drives to improve their performance and effeciency. At this time, the motor load is primarily DOL; however, in the near future it is expected that VFD will make up a signifinct share. VFD motors provide the distinctive advantage of being able to operate over a wider range of speed whilst maintaining a constant maximum torque, given by (3).

$$
T_{\max }=\frac{K\left(\frac{V}{f}\right)^{2}}{\frac{R_{s}}{f} \pm \sqrt{\left[\left(\frac{R_{s}}{f}\right)^{2}+4 \pi^{2}\left(L_{s}+L_{r}^{\prime}\right)^{2}\right]}}
$$


In (3), $V$ is the applied voltage, $f$ is the supply frequency, $L_{s}$ and $L_{r}$ ' are stator and stator referred inductances, $R_{s}$ is the stator resistance and $K$ is a constant. In cases where $f$ is not too low, $R_{s} / f<<2 \pi\left(L_{s}+L_{r}{ }^{\prime}\right)$ and the equation reduces to (4).

$$
T_{\max }= \pm \frac{K\left(\frac{V}{f}\right)^{2}}{2 \pi\left(L_{s}+L_{r}^{\prime}\right)}
$$

It can be seen from (4) that maintaining a constant $V / f$ ratio helps the rotor to develop a constant maximum torque which allows for a constant torque mode of operation (except at low speeds). The impact of $R_{s} / f$ cannot be neglected at low frequencies which are bound to affect the motor maximum torque which is of particular interest for the sub-synchronous range of frequencies that may appear at the motor terminals in case of SSR phenomenon. It is noted that in order to achieve high torque at low speeds or during motor starting, a relatively high $V / f$ ratio needs to be maintained.

The drives have been considered to have passive front ends (diode bridges) as active front ends (GTOs/ IGBTs) are rare due to their high overall cost Variable voltage and frequency for motor drive control has been realized through Voltage Source Inverter (3-phase, 6-pulse VSI) using SPWM (Sinusoidal Pulse Width Modulation) switching. SPWM technique is widely used for generating sinusoidal inverter output voltage without lower-order harmonics. Higher order harmonics that may be introduced have been eliminated using filters. Static Power Converter within the motor drive unit has not been modelled with any in-built current controller or limiter. Details about the EMT model for the PWM inverter and 6-pulse bridge rectifier (passive front end) can be found in [16]

Two common VFD control designs e.g. open loop and closed loop have been investigated for torsional interactions during SSR phenomenon. .

a) Open loop control:The control strategy implemented compares the motor terminal frequency $\left(f_{s}\right)$ with a reference set point $\left(f_{\text {setp }}\right)$ and passes the corresponding frequency error $\left(f_{\text {error }}\right)$ through a PI controller, as shown in Fig. 2.

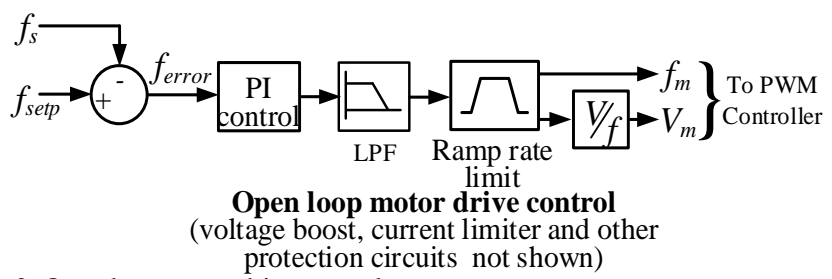

Fig. 2. Open loop motor drive control

The output of the PI block is passed through a low pass filter (to remove any unwanted noise) and then through a ramp rate limiter to restrict the rate of change of motor speed. This is particularly important for passive front end drives as they do not support slip power recovery causing any power if fed back to the drive to get wasted in a dump load. The output of the rate limiter is passed through a $V / f$ block to vary the inverter ouput frequency and voltage simultaneously while keeping the ratio constant such that maximum electromagnetic torque can be achieved throughout the speed range.

b) Closed loop control: Closed loop VFD control strategy implemented for the study explicitly keeps track of the motor speed by measuring it at the shaft $\left(w_{r}\right)$ and comparing it to a reference setpoint $\left(w_{\text {rsetp }}\right)$ to get the motor speed error $\left(w_{\text {error }}\right)$, as shown in Fig. 3. An additional slip speed $\left(w_{\text {slip }}\right)$ control loop is introduced to maintain the motor slip within a specified value (e.g. $2 \%$ ). Whenever the motor speed exceeds this value the motor speed error is updated to a new value ( error $^{*}$ ) which is then passed through the PI controller to get the inverter set points for switching pulses while keeping the $V / f$ ratio constant [17]. Closed loop VFD control is used for process control applications requiring precise motor speed control.

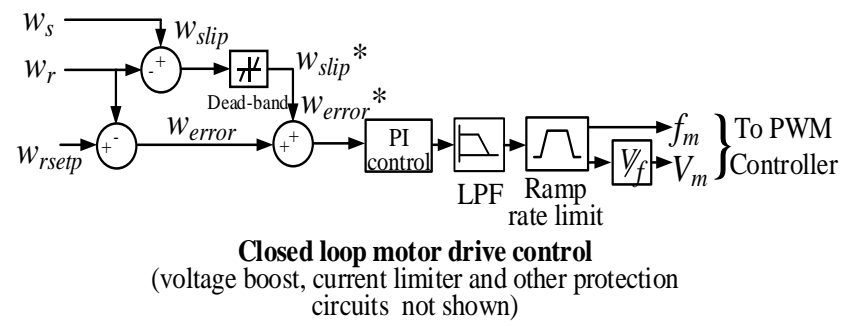

Fig. 3. Closed loop motor drive control

c) Performance Comparison of Open and Closed loop drive controls: As discussed already, both type of motor controls maintain a fixed $V / f$ ratio. However, this is maintained only till the nominal operating point of the motor. Above this point $V / f$ ratio is no longer maintained as the motor terminal voltage is restricted to its nominal value in order to avoid insulation damage.

Operation of the VFD motor controls is shown in Fig. 4 by simulating a step disturbance in the load torque (Fig. 4 (a)). As open loop control has no speed feedback, the motor slows down slightly (Fig. 4 (d)) to cater to the increased torque demand by increasing its slip (Fig. 4 (c)) and thereby drawing more power (Fig. 4 (b)). Closed loop control, on the other hand, is seen to have successfully maintained the motor speed (and slip) by changing the supply frequency at the motor terminals in order to cater to the new load power requirement.
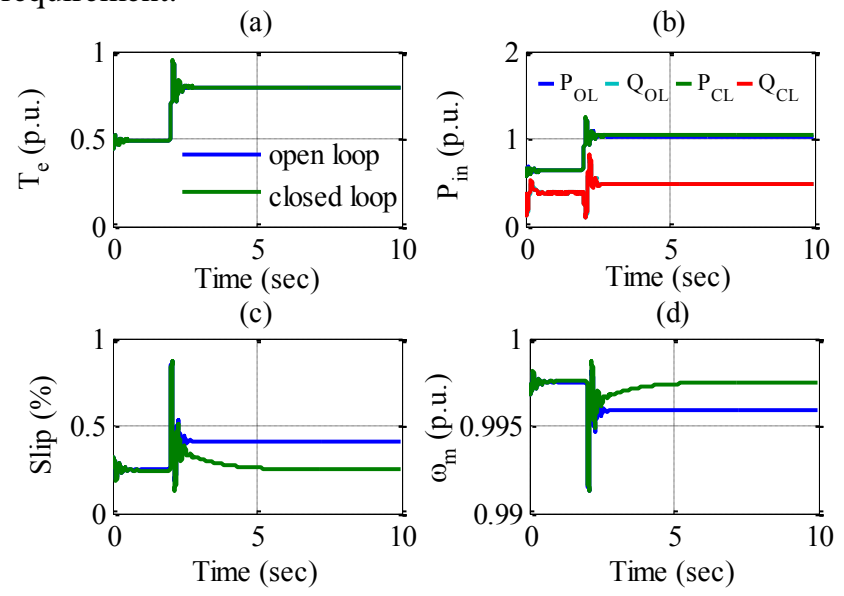

Fig. 4. Open and closed loop VFD motor control 


\section{Method adopted for Aggregation of Motor Load at the Bulk Transmission Level}

Induction machines constitute a major portion of the overall power system load, making their accurate representation in simulation studies very important for realistic reproduction of system dynamic response. Motor loads connected at a particular bus will practically include different types of induction machines (e.g. small, large industrial motors), each having a different dynamic characteristic. It is thus appropriate to model this diverse group of machines by an aggregated equivalent motor, as given in [4]. Aggregation method $\mathrm{B}$ is used here to combine the $n$ motors at a bus into a single equivalent motor. These $n$ motors are made up of equal proportions of small and large motors. The equivalent motor inertia is calculated as the weighted average of the individual motor inertias in the group. The weighting co-efficient, $\sigma$, is defined as the relative KVA rating of the individual motor with respect to the KVA rating of the aggregate motor:

$$
\sigma_{j}=\frac{K V A_{j}}{\sum_{j=1}^{n} K V A_{j}}=\frac{K V A_{j}}{K V A_{a g g}}
$$

The electrical parameters of the aggregate motor have been calculated using a weighted average of branch admittances i.e. stator, rotor and magnetizing branches, as in (6). This is equivalent to having multiple motors connected in parallel.

$$
\frac{1}{Z_{a g g}}=\sum_{j=1}^{n} \frac{\sigma_{j}}{Z_{j}}
$$

Also, the critical slip (slip at which the motor torque-speed characteristic is the maximum) of the equivalent motor is obtained by calculating the weighted average of the individual motor slips. This ensures that the operating region of the aggregate motor lies between the regions of the individual motor characteristics.

\section{CASE STUdiES}

\section{A. Study Network}

The network under study is based on the IEEE First Benchmark model. A schematic diagram of the network is presented in Fig. 5. It consists of a single machine connected radially to a series capacitor. Rest of the network is represented by a voltage source. A load center is considered to be in close electrical proximity and represented by an aggregate load bus. Four different types of loads are considered: a) constant impedance; b) constant current; c) direct on-line motor and d) drive based motor load. Constant impedance and current type loads comprise the aggregated static load while DOL and VFD motors represent the aggregated dynamic load. The VFD motors have been aggregated at $11 \mathrm{kV}$ as majority of the large drive based industrial loads are connected at this voltage level. Rest of the loads are aggregated at the $500 \mathrm{kV}$ level. The VFD motor drive has been modelled in detail with the dc bus reactor and capacitive filter tuned appropriately for continuous conduction of current. The system dynamic response is naturally unstable with $60 \%$ series compensation due to SSR phenomenon, without consideration of any load. All the case studies have been carried out at this compensation level.

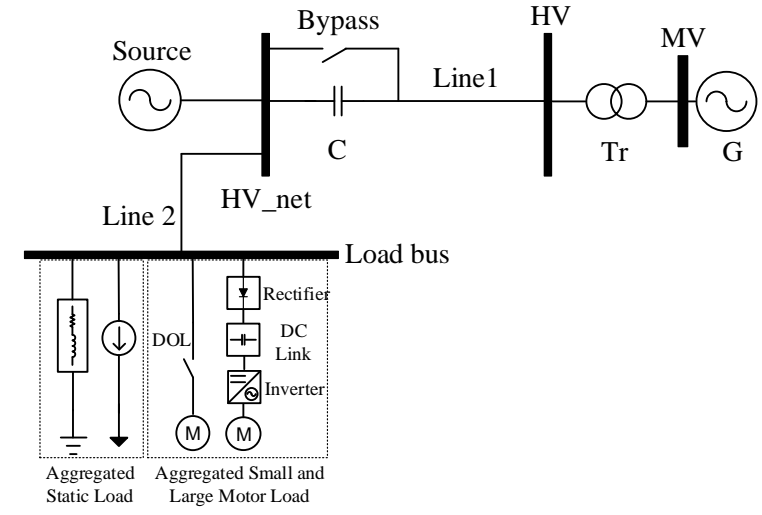

Fig. 5. Schematic of network under study

\section{B. Impact Of Load Modelling On Damping Of Torsional Oscillations}

In this study, SSR oscillations are monitored in the turbine output power for different load compositions at the load bus in order to investigate how realistic the classical assumption of either neglecting the loads completely (e.g. in IEEE $1^{\text {st }}$ and $2^{\text {nd }}$ Benchmark models for SSR studies) or modelling them as constant impedances is. Results presented in Fig. 6 clearly indicate that including load dynamics may actually modify the effective damping for torsional interactions to an extent where the response may actually change from being unstable for Type 1 and 2, to being stable for Types 3, 4, 5 and 6. Such conservative results obtained using classical load models may have significant impact on decisions made at the planning stage (regarding location and/or degree of compensation) and during operation (setting improper thresholds for online alarms against dangerous interactions).

Table I describes the different load types that have been considered for the study. These load compositions are not necessarily realistic, but do provide a basis for emphasizing the importance of dynamic load modelling in SSR studies.

Table I

List of load types considered

\begin{tabular}{|c|l|}
\hline Load Type & \multicolumn{1}{|c|}{ Description } \\
\hline Type 1 & Loads neglected \\
\hline Type 2 & $100 \%$ Const. Impedance \\
\hline Type 3 & $50 \%$ DOL and 50\% VFD based Motor loads \\
\hline Type 4 & $100 \%$ DOL connected Motor load \\
\hline Type 5 & $\begin{array}{l}\text { 30\% Const. Impedance 30\% Const. Current 40\% DOL } \\
\text { connected Motor load }\end{array}$ \\
\hline Type 6 & $50 \%$ Constant Impedance 50\% DOL connected \\
\hline
\end{tabular}

Fig.6 shows that the nature and composition of loads produce markedly different damping responses. While representing downstream loads as constant impedance type may result in slightly improved damping response compared to when loads are completely neglected, it is still a conservative assumption of the true nature of the damping provided for torsional oscillations in SSR studies. Type 5 and type 6 represent the more realistic grid condition (at present scenario) and are found to provide the best damping among the six different load types that have been considered. 


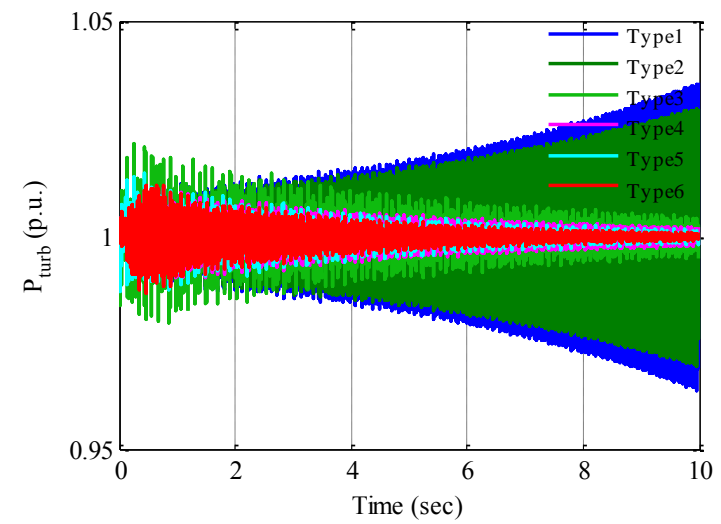

Fig. 6. Damping comparison of different load model

This case study clearly reveals the importance of accurate load representation for the reliable assessment of system dynamic performance and precise assessment of SSR risks, especially when the load center is close to generation.

\section{Impact Of Motor Size And Location On Damping Of Torsional Oscillations}

Section B briefly explored how varying the load model used for simulations of SSR will influence the damping of torsional oscillations. The inclusion of motor load was found to have the most significant impact. This section will study how the location, inertia (i.e. motor size in terms of rotational mass or moment of inertia, $J$ in $\mathrm{kgm}^{2}$ ) and composition of the motor load will impact the effective damping. The parameters used for the motor load can be found in [15].

Fig. 7 (a) shows the generator turbine output power $\left(P_{\text {turb }}\right)$ and power flow through series capacitor $\left(P_{c a p}\right)$ for two different locations (load bus and HV bus) of DOL type motor load $\left(J_{a g g}=39027 \mathrm{kgm}^{2}\right)$ during SSR. It can be seen that when the aggregated motor load is installed at the HV bus it provides much better damping than when it is installed at the load bus. This can be justified by monitoring the power flow through the series capacitor $\left(P_{c a p}\right)$. Fig. 5 shows that when loads are connected at the load bus, total power from the generator $(\mathrm{G})$ has to flow through the series capacitor (C) as opposed to when loads are connected at the HV bus. As the generator is feeding into the SSR oscillations and the capacitor is exchanging energy with it during this period, higher power flow through the capacitor negatively affects the damping contribution from loads.

However, Fig. 7 (b) illustrates that the opposite is true if the inertia of the motor load is reduced. An aggregate motor with the same demand but reduced inertia $\left(J_{a g g}=18212 \mathrm{kgm}^{2}\right)$ provides more damping when it is installed at the load bus than it does when installed at the HV bus.

The low inertia motor provides positive damping at both the locations. However, at the HV bus it causes large oscillations in the turbine output power as well as the power flow through the capacitor. These large oscillations are caused by the proximity of the small motor to the source of the disturbance $(\mathrm{G})$. The number of testing locations is limited for this simple radial network. The results presented here emphasize the importance of load location and size in determining the effective damping contribution and further study, in a more complex system, will be required to fully understand this variation in damping.
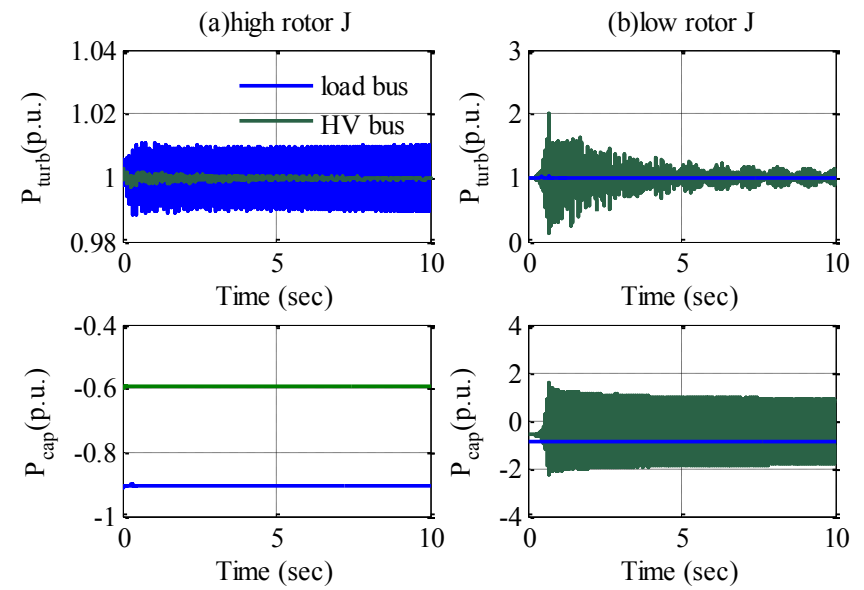

Fig. 7. Impact of motor shaft inertia and motor location on SSR damping. For the high inertia motor $J_{a g g}=39027 \mathrm{kgm}^{2}$ and for low inertia it is $18212 \mathrm{kgm}^{2}$

The load bus is a more realistic load location for this study network. It is used for further analysis of why an equivalent motor with lower inertia can provide better damping than one with higher inertia. Fig. 8 shows that the lower inertia motor is more responsive and the rotor speed oscillations closely follow the variations in system frequency. For maximum damping the change in speed (and hence active power consumption) of the motor should be in phase with the change in system frequency. However, the location of the load is also an important factor, as proximity to generation may cause higher oscillations and poorer damping from the same motor.

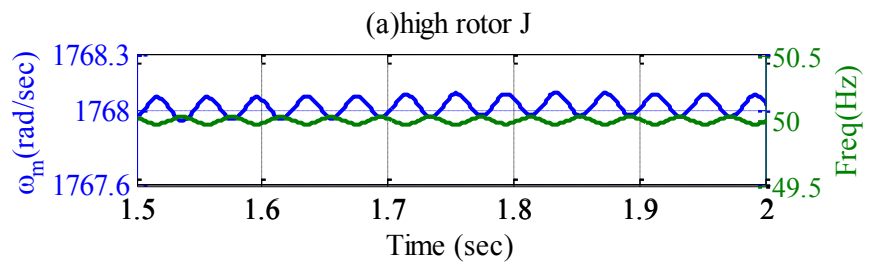

(b) low rotor $\mathrm{J}$

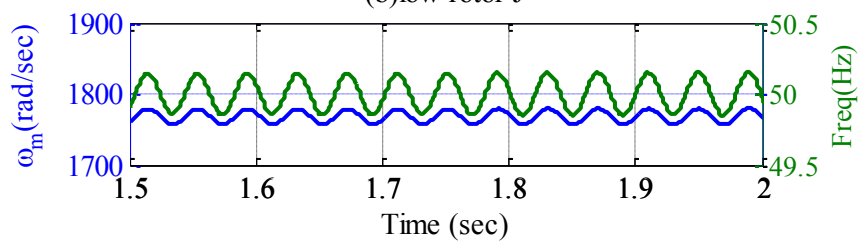

Fig. 8. Phase relationship between motor speed and bus frequency when a high inertia (a) and low inertia (b) motor loads are connected at the load bus

\section{DOL Type Motor Interaction With SSR}

Three-phase induction motors are the most common industrial machines in operation. They can have different types of starting and interfacing techniques like Direct-OnLine (DOL), star-delta transformer, autotransformer or variable speed drives. Based on the information availabe from the vendors of motor drives in Great Britain almost $80 \%$ of the total industrial and service sector motor loads are DOL type. For a DOL start motor, the stator is directly connected to the supply, making them naturally vulnerable to any voltage and/or frequency fluctuations at their terminals.

During SSR, subsynchronous frequency current components flow into the network. These components give rise to a stator flux that rotates at a speed lower than the 
synchronous speed. The overall stator flux magnitude is then determined from the superposition of the fundamental $\left(\psi_{s}\right)$ and the low frequency $\left(\psi_{f}\right)$ components. As the magnetic field oscillates between $\psi s+\psi f$ and $\psi s-\psi f$, induction machine torque (hence speed) starts to oscillate. Fig. 9 shows the turbine output power $\left(P_{\text {turb }}\right)$ and the induction motor electrical $\left(T_{e}\right)$ and mechanical $\left(T_{m}\right)$ torque oscillations along with the respective FFT results. A single cycle three phase short circuit fault is simulated in this case study to magnify the impact of generator shaft torsional oscillations on $T_{e}$. The induction motor is connected at the load bus.

(a)
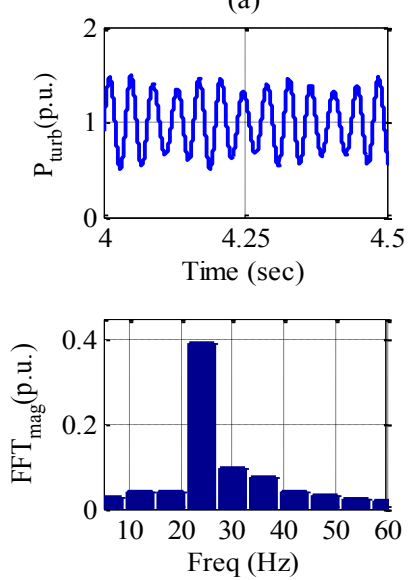

(b)
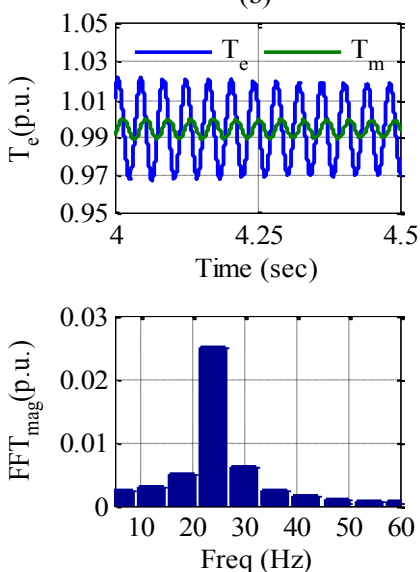

Fig. 9. DOL type induction motor interaction with SSR

Fig. 9 (b) shows that the same frequency component appears in the oscillations in both $T_{e}$ and $P_{t u r b}$ (fixed mechanical torsional frequency of $25 \mathrm{~Hz}$ ). As electrical proximity between the generation and load centre increases, these interactions become particularly important. This phenomenon can be termed as Subsynchronous Resonance Load Interactions (SSR-LI), which is distinct from the three already reported interactions, namely SSR, SSTI (Subsynchronous torsional interaction) and SSCI (Subsynchronous Control Interaction). SSR-LI involves interaction between the generator shaft, network LC mode and dynamic load. Oscillations appearing in the electrical torque cause a change in the operating point of the motor

(a)
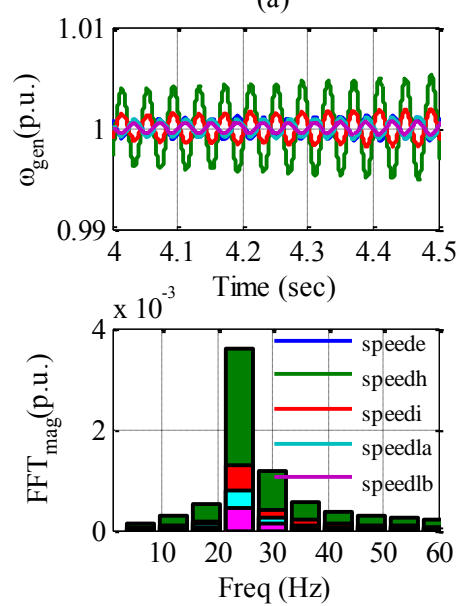

(b)
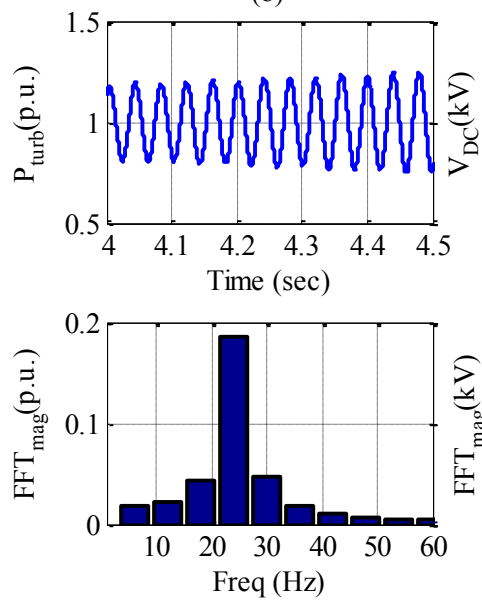

every subsynchronous cycle, leading to significant stress on the motor shaft. Oscillations seen in the armature current may further cause over-heating/ over-fluxing problems for the motor. This type of interaction may prove to be particularly detrimental for constant torque type loads (e.g. conveyers, positive displacement pumps, hoists) [18]. Thus, the presented results show that SSR can not only interact with generator shafts but can also interact with motor shafts and interfere with the normal operation of motor loads.

\section{E. VFD Based Motor Interaction with SSR}

The main objective of this case study is to investigate how the SSR oscillations propagate through power electronic intefaces and interact with the motor shaft. Fig. 10 (a) presents speed oscillations at different points of the generator-turbine shaft, i.e. exciter (speede), high pressure turbine (speedh), intermediate pressure turbine (speedi), low pressure turbine stages A and B (speedla,speedlb), along with corresponding FFT results. Fig. 10 (b) presents the oscillation in the turbine output power $\left(P_{t u r b}\right)$ and its correponding FFT. Fig. 10 (c) presents the dc link capacitor voltage $\left(V_{D C}\right)$ of the VFD and its FFT, which indicates that the torsional oscillations can pass through the diode bridge to appear on the dc side and may then propagate to the motor side through the VSI. Fig. 10 (d) shows that this oscillation reflects on to the machine electrical torque $\left(T_{e}\right)$ which starts oscillating across the mechanical load torque $\left(T_{m}\right)$ of the motor. It is interesting to see a $25 \mathrm{~Hz}$ frequency component appearing at the motor terminal in additon to the $5^{\text {th }}$ and $7^{\text {th }}$ harmonics that are characteristic of the 6-pulse inverter. The values appearing at the motor drive end have been presented in absolute terms as their magnitude is significantly lower than the generator shaft oscillations. The magnitude of the oscillations in $T_{e}$ depends on the dc link capacitor (filtering). However, as the capacitor is tuned to smoothen out dc voltage ripples it may not be able to effectively filter out subsynchronous frequecies. Sustained oscillations in $T_{e}$ may result in rotor shaft damage.

The results clearly indicate that SSR can translate through passive front-end VFD drives, for both type of controls. The results presented here are for closed loop control only.

(c)
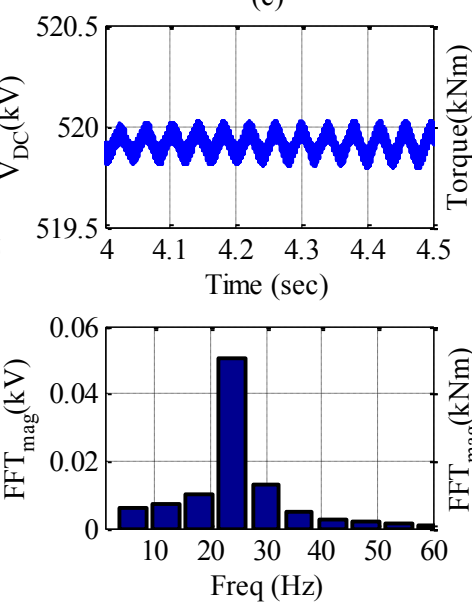

(d)
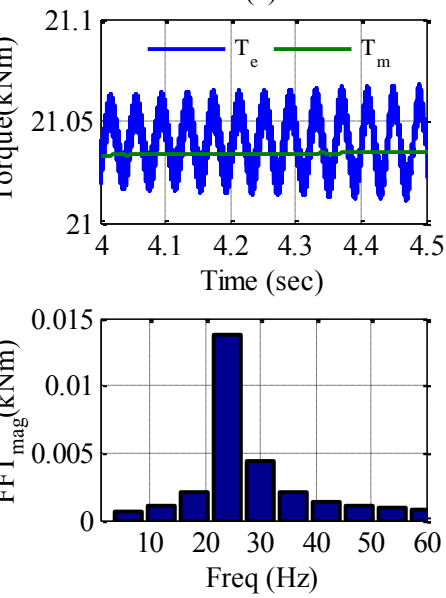

Fig. 10. (a) Generator-turbine shaft speed, (b) Turbine output power, (c) VFD dc link capacitor voltage and (d) Motor torque 


\section{FUTURE WORK - AUXILIARY DAMPING CONTROL}

A large number of SSR mitigation techniques are in use within the industry, e.g. Static Blocking Filters (BFs), Supplementary Excitation Damping Control (SEDC), Damping Rotor Resistor, TCSC, HVDC controls [19]. However, each of these solutions suffers from certain shortcomings, e.g: BFs are passive filters and once tuned cannot be operated optimally in real-time and need significant capital investment; introducing additional damping resistance in the rotor winding increases the complexity of rotor construction and must be done at the manufacturing stage; and TCSC or HVDC controls are promising but quite expensive.

The results presented here indicate that induction motor loads can positively contribute towards torsional damping. Therefore, it is proposed that a certain amount of DOL start induction motor loads may be paid to be in service at specific locations (e.g. as ancillary service or demand side management for torsional damping) alongside VFD motor loads equipped with auxiliary damping controllers. Efficient and robust auxiliary damping controller may be designed by making subtle modifications to the existing VFD control loop. This can provide additional damping at very minimal cost. Auxiliary power plant loads like Forced Draft (FD), Induced Draft (ID) fans present an attractive option as they are electrically close to generators and are large, $11 \mathrm{kV}$ VFD induction motor loads. Smaller motor loads that run downstream can be included to extract damping in large interconnected networks. Future work involves the design of an auxiliary damping controller using existing VFD motor loads, fully understanding the sensitivity to location and inertia, and studying the effectiveness of the proposed demand side solution for providing additional damping of SSR oscillation for meshed power systems.

\section{CONCLUSION}

This paper investigates the impact of dynamic load modelling on EMT studies of SSR. Loads are classically either neglected or modelled as constant impedances for SSR studies. The objective of this work is to investigate this classical assumption by modelling different types of loads (aggregated at the bulk transmission level) to highlight their relative impact on the damping of torsional oscillations.

Analysis shows that static load models give conservative results and the modelling of dynamic loads is of critical importance in order to capture system dynamics or transient behavior for SSR studies. Dynamic loads have been modelled as induction motors (DOL and drive based). Both open and closed loop VFD motor controls have been investigated. Case studies highlighting the impact of size and location of motor type loads in providing damping for torsional oscillations have been presented in detail. Results reveal that low inertia motors have the capability to provide better damping than high inertia motors because their speed oscillations closely follow subsynchronous frequency oscillations. However, the load location plays a particularly important role in determining the damping, as it affects the power flow through the series capacitor.

The phenomenon of Subsynchronous Resonance Load Interaction (SSR-LI) for conditions where the load centers are electrically close to the generation centers has been introduced and discussed for both DOL and VFD based dynamic motor loads.
Results have also been presented to show the propagation of torsional oscillations across passive front end VFD motor loads and the negative impact of subsynchronous components appearing at the motor terminals has been discussed in terms of motor operation. Finally, the potential use of VFD motor drives for mitigation of SSR through supplementary damping control is proposed as an avenue for future research.

\section{REFERENCE}

[1]. J. Milanovic, A. Adrees, "Identifying Generators at Risk of SSR in Meshed Compensated AC/DC Power Networks", IEEE Transactions on Power Systems, Vol. 28, No. 4, May 2013.

[2]. IEEE Task force for Load Representation for Dynamic Performance, "Load Representation for Dynamic Performance Analysis", IEEE Transactions on Power Systems, Vol.8, No. 2, May 1993

[3]. O.H. Abdallah, M.E. Bahgat, A.M. Serag, M.A. El-Sharkawi, "Dynamic Load Modelling and Aggregation in Power system Simulation studies", Power systems conference, Middle-East, MEPCON, 2008

[4]. Farhad Nozari, M. David Kankam \& William W. Price, "Aggregation of Induction Motors for Transient Stability Load Modeling," IEEE Trans on Power Systems, Vol. PRWS-2, No. 4, 1987.

[5]. B.L. Agarwal, J.A. Demcko, R.G. Farmer and D.A. Selin, "Apparent Impedance Measuring System (AIMS)," IEEE Transactions on Power Systems, Vol. 4, No. 2, May 1989

[6]. Dong Han, Guoqiang Zhang, Tao Lin and Hui Ding, "The effect of load models on electromagnetic transient stability in AC/DC power systems", Electricity Distribution (CICED), 2012 China International Conference, pp. 1-4, 2012

[7]. E. Sorrentino, O Salazar and D. Chavez, "Effect of generator models and load models on the results of the transient stability analysis of a power system", in Universities Power Engineering Conference (UPEC), pp. 1-5, Proceedings of the $44^{\text {th }}$ International, 2009

[8]. E. Santos, T.I. Asiain, D. Ruiz and D. Olguin, "The effect of load chracteristics on the transient stability studies of a laboratory electric power system including induction generators", International Conference on Electric Power Engineering, PowerTech Budapest 99, pp. $-81,1999$

[9]. W. Mauricio and A. Semlyen, "Effect of Load characteristic on the Dynamic Stability of Power Systems", IEEE Transactions on Power Apparatus and Systems, Vol. PAS-91, Issue no. 6, pp. 2295-2304, 1972

[10]. Chen, W.H.; Bi, T.S.; Fang, J.Y.; Yang, Q.X., "Interaction of torsional dynamics and loads," in Advances in Power System Control, Operation and Management (APSCOM 2009), 8th International Conference., pp.1-5, 8-11 Nov. 2009

[11]. P. Kundur, Power System Stability and Control. New York: Mc-GrawHill, 1994.

[12]. Regulski, P.; Vilchis-Rodriguez, D.S.; Djurovic, S.; Terzija, V., "Estimation of Composite Load Model Parameters Using an Improved Particle Swarm Optimization Method," in Power Delivery, IEEE Transactions on, vol.30, no.2, pp.553-560, April 2015

[13]. J.V. Milanovic, K. Yamashita, S.M. Villanueva, S.Z. Djokic, "International Industry Practice on Power System Load Modeling," IEEE Transactions on Power Systems, vol. 28, pp. 3038-3046, Dec. 2012.

[14]. Department of Energy and Climate Change (2014). Energy Consumption in the UK Service sector data tables 2014 update. [Online]. Available: https://www.gov.uk/government/statistics/energyconsumption-in-the-uk

[15]. IEEE Task Force on Load Representation for Dynamic Performance, 'Standard load models for power flow and dynamic performance simulation,' IEEE Trans on Power Systems, Vol. 10, No. 3, 1995, pp. 1302-1313.

[16]. DigSILENT Downloads, Technical References [Online]. Available: http://www.digsilent.de/index.php/downloads.html

[17]. A. Hughes and B. Drury, Electric Motors and Drives, 4th ed. Elsevier Ltd., 2013.

[18]. Schneider S.A. (1995, May). Adjustable Frequency Controllers. [Online].Available:http://www2.schneider-electric.com/resources /sites/SCHNEIDER_ELECTRIC/content/live/FAQS/105000/FA10538 7/en_US/SC100.pdf

[19]. Padiyar, K. R. Analysis of subsynchronous resonance in power systems. Springer Science \& Business Media, 2012. 